\title{
Custom-made reverse shoulder arthroplasty for severe glenoid bone loss: review of the literature and our preliminary results
}

\author{
G. Porcellini ${ }^{1}$, G. M. Micheloni ${ }^{1 *}$, L. Tarallo ${ }^{1}$, P. Paladini ${ }^{2}$, G. Merolla ${ }^{2}$ and F. Catani ${ }^{1}$
}

\begin{abstract}
The treatment of severe glenoid bone loss in shoulder arthroplasty represents a challenge, and the results of current prosthetic designs with only glenoid fixation still remain unsatisfactory. In the past decade, customized glenoid prostheses have been developed to address severe glenoid arthritis and in the revision setting. In this review, we analyzed the current surgical options, the classification limits, past literature evidence, and our preliminary results of 6 patients ( 3 male, 3 female) treated with a reverse implant and custom-made glenoid implant (ProMade; LimaCorporate, Italy). Computer analysis of the residual shape and the amount of glenoid bone stock in association with new classifications could help the surgeon to obtain good clinical and radiological outcomes. The development of navigation systems could improve the adequacy of the implant and, thus, the reliability and longevity of the implant itself.
\end{abstract}

Keywords: Severe bone loss, Custom-made implant, Reverse shoulder arthroplasty, Bone stock

\section{Introduction}

The management of glenoid bone loss is a highly demanding challenge for orthopedic surgeons, and the number of cases is expected to rise in the future, in proportion to the increase of prosthetic implants, life expectancy, and functional demand of patients [1-3].

The main causes of bone defects include degenerative bone diseases of the glenohumeral joint, chronic dislocations, congenital malformations, sequelae of autoimmune diseases including rheumatoid arthritis, status post proximal humerus fractures treated with fixation devices (intramedullary plates or nails) secondary to damaging of the glenoid articular surface due to extrusion or migration of fixation devices [4]. Further recurrent reasons are related to previous prosthetic implant complicationsseptic or aseptic mobilization of the glenoid component in anatomical or reverse shoulder implants frequently induces a significant loss of glenoid bone stock, forcing

\footnotetext{
*Correspondence: gianmario.micheloni@libero.it

${ }^{1}$ Department of Orthopaedic Surgery, University of Modena and Reggio

Emilia, Modena, Italy

Full list of author information is available at the end of the article
}

the surgeon to make extremely difficult decisions in order to guarantee the best compromise between functionality and pain regression.

Glenoid bone loss can be mild (B2 and B3 according to Walch classification) and is usually treated with eccentric glenoid reaming, hemiarthroplasty, bone grafts, and augmented implants [5-8]; or it could present significant concentric or eccentric defects $[6,9]$.

The definition and quantification of severe bone loss of the vault and the glenoid surface are controversial. In our experience, we consider a case to be 'severe' if it is impossible to treat with traditional implants including the use of wedges.

\section{Classifications}

Various classifications are present in the literature, but the most commonly used are the classifications proposed by Antuna and Seebauer.

Antuna et al. classified glenoid bone loss as central, peripheral, and combined, with each group being partitioned into mild, moderate and severe [10].

Seebauer et al. classified the defects as centric and eccentric erosions. Centric defects (contained) are 
shallow (C1), deep (C2), cavitary (C3) or destructive (C4), whereas eccentric defects are further partitioned based on size and location [4].

The above-mentioned classifications are particularly useful to describe the defects, although less beneficial in terms of treatment and surgical plan.

In severe combined defects (according to Antuna classification), or in C4 and E4 defects (according to Seebauer classification), the decision-making process is challenging.

Identifying a new classification system for severe bone loss based on a three-dimensional (3D) reconstruction computed tomography (CT) scan (according to Seebauer classification), residual bone quality and shoulder stability could be useful to obtain common, shareable, and reliable surgical treatment solutions. The aim of this classification is to find cases that are potentially treatable with traditional implants and cases requiring custommade implants.

\section{Surgical treatments}

Glenoid bone loss can be treated with reverse shoulder arthroplasty (RSA) and bone grafting. These techniques allow the management of mild-to moderate defects with autologous bone or titanium wedges/hemi-wedges placed on the basis of bone loss morphology and extension.

Boileau et al. proposed the bony increased offset (BIO) technique, with compression of the bone graft by the implant to achieve a more favorable environment for graft incorporation. The main goal of this technique is to achieve lateralization of the glenohumeral center of rotation. The size and morphology of the bone graft are determined by the extent of the bone defect, the joint line, and soft-tissue tensioning [11]. Boileau et al. reported encouraging outcomes with a graft incorporation rate of $98 \%$ in a study of 42 patients treated with BIO-RSA, although the major criticism was related to the risk of graft non-union and graft reabsorption [12]. In our opinion, the management of glenoid retroversion could become challenging with this surgical solution. Additionally, eccentric bone grafts show a lower union rate compared with concentric grafts as recently reported in the literature [13].

Other authors have suggested augmented glenoid implants to restore the anatomic joint line and minimize the non-union risk [14]. Studies on early metal-backed wedge-shaped glenoid augments in anatomic implants reported a high failure rate after a 10-year follow-up evaluation $[8,15]$. Recently, the introduction of trabecular metal components has renewed the interest in these types of implants with excellent early outcomes [16].

The major difficulties are in the management and treatment of severe glenoid bone loss, which are unmanageable in cases of the above-listed prosthetic implants. Some authors suggest the use of endoprosthesis, although various studies in the literature have shown highly scarce and mediocre clinical outcomes when compared with the use of total shoulder replacement [17-22]. Other authors have suggested the use of bone grafting, although there is also insufficient evidence of clinical outcomes and many critical aspects to report, i.e., the demanding surgical technique and the potential risk of bone reabsorption over time and, hence, mobilization of the implant itself [7, 18, 23-29].

\section{Custom made-implants}

Indications for custom-made reverse implants are related to the patient's functional demand and to the remaining bone stock. Custom-made implants are not recommended in patients with scarce or reduced functional demand, considering the elevated costs and not yet standardized results.

A flat glenoid metal back, in the case of severe bone stock, has an extremely low sitting percentage and the central peg does not ensure adequate stability.

A minimum vault depth of $10 \mathrm{~mm}$ to achieve initial fixation and a volume that allows the insertion of a minimum of two screws is reported in the literature [30]. Furthermore, there is the consensus that implant stability is achieved if the peg is almost $50 \%$ in length into the glenoid bone [31].

The use of 3D reconstruction CT scans, with specific metal artifact reduction (MAR) software, associated with the development of custom-made implants in orthopedic surgery, provides interesting solutions for cases of severe glenoid bone loss.

Progress in 3D printing technology has led to the manufacture of an implant that matches, as accurately as possible, the glenoid deformity [32].

Patient-specific instrumentation (PSI) improves the accuracy of component implantation as planned in the preoperative analysis.

The advantages of custom-made surgery include the possibility to determine the stability on elements that are still intact (i.e., the spine of the scapula or the coracoid process), to fill the gap in the bone and to adequately reconstruct the original joint line, to place the fixation devices (screws) with the proper length, and to determine the best orientation (Fig. 1). This technology is based on preoperative CT scans and analyzed by computerized systems able to identify the bone loss entity and to determine the fixation elements in which the glenoid component of the implant is to be placed. 


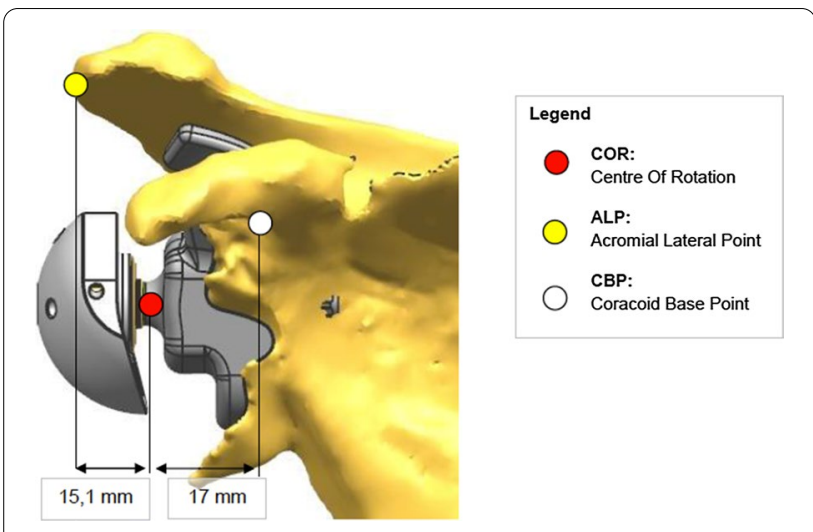

Fig. 1 Center of rotation and landmarks for joint line reconstruction

\section{Preoperative Assessment and Computer-Assisted Design/Computer-Assisted Manufacturing (CAD/ CAM) analysis}

Anatomical reconstruction, and evaluation of the patient's image data congruency and quality are performed during the initial phase (i.e., congruence of anatomical site, opportunity to locate and define anatomical landmarks, slice thickness, presence, and entity of metal artifacts, 'loose' bone areas, areas to be removed or to be preserved), consistently with the selected surgical technique and implant insertion direction.

Anatomical reconstruction is performed using commercially available Materialize Mimics Suite software.

It is essential that the evaluation is performed on the different areas of the scapula and humerus, including determining the impact of the above-mentioned parameters on the geometrical precision, the possible design, and the surgical technique.

In each case, the design is directed to optimize the metal-bone interface area, the primary stability, and the load transfer, with metal engagement on the most supportive areas, with an ultimately consecutive phase of bone remodeling (drilling, curettage, reaming, highspeed burr).

In revision cases, it is frequently essential to compromise on bone sparing and bone removal in the surgical technique, since it is commonly related both to metal artifacts and to uncertainty of bone loss because of the hardware removal phase.

A wrong estimation of the bone loss/bone not visible (due to artifacts or revision procedure) could lead to a loose custom implant or an extremely tight-fitting implant and manual preparation would therefore be required.

Thus, in order to overcome this situation, planning an adequate guided drilling/reaming and determining the reliable landmarks and the stable bone areas could allow a proper fitting in the above-mentioned cases.

The design of the implant will have to allow primary stability, recovery of biomechanical balance, and adequate range of motion.

A 3D reconstruction facilitates specific planning of the features of the implantable component, in addition to patient-specific instruments and surgical technique, essential for cautious and guided preparation of the site and to identify the anatomical landmarks on the patient's anatomy and on anatomical replicas.

Frequently, custom glenoid implants are used when a standard implant would not allow adequate stability or stress distribution. Hence, to overcome the limitation of conventional implants, customization facilitates an increase of the fixation site, progressively growing implant invasiveness toward the coracoid process, increasing peg dimension or length, uncoupling peg direction with glenosphere coupling direction, adding localized screws or, in other cases, stabilizing the system with acromial support pads and flanges.

The best production process is evaluated in order to optimize the material shape and performance during the design phase. For instance, maximizing the use of high integration surfaces in contact with native bone (in the case of soft-tissue reattachment sites although limiting the presence of abrasive surfaces or cutting edges in which soft-tissue sliding over implant is noted), maximizing flexible structures that could permit to fill the gaps or transfer loads from the implant to the bone effortlessly, allowing the required resistance to the supportive structures.

Design and CAM preparation are prepared by means of the CAD system (Siemens NX Suite, solid modeling and $\mathrm{CAM}$ ) in order to allow consistent and precise preparation of the 3D models for additive manufacturing and standard production processes, where precision is required. The use of non-technical 3D visualization and interaction tools are fundamental in order to permit the surgeon to evaluate the $3 \mathrm{D}$ reconstruction and the anatomy/implant/instrument interaction, essential for the challenging analysis of highly deformed anatomies, frequently associated with custom cases.

\section{Preliminary experience and results}

Six patients (3 male, 3 female) treated with a reverse implant and custom-made glenoid implant (ProMade; LimaCorporate, Italy) at the Ospedale Cervesi of Cattolica (Rimini, Italy) and at the Policlinico of Modena (Modena, Italy) were included and evaluated in our case study.

The mean age of the patients at the time of the surgical procedure was 64 years (minimum 48 and maximum 74 years) and the mean follow-up time was 31.67 months 
(minimum 25 and maximum 38 months). A case of painful partial replacement in status post previous surgical procedures, a case of an infected anatomical implant, a case of status post scapular fracture in a patient with rheumatoid arthritis, a case of status post fracture treated with the open reduction internal fixation technique, two cases of mobilization of reverse implant in status post previous surgical procedures.

According to Antuna classification, each case presented severe combined bone loss and according to Seebauer classification, four cases were $\mathrm{E} 4$ and two cases were $\mathrm{C} 4$.

Each of our patients underwent preoperative clinical evaluation (Constant score, ASES score, VAS scale), radiological assessment and 3D CT scan with specific MAR software (Fig. 2a-d).

The CT scan for each case was presented to LimaCorporate in order to analyze the size and shape and to select the most adequate fixation elements to obtain stability of the glenoid component.

The LimaCorporate Company provided the orthopedic surgeon with a file containing images, information, an accurate description of the entire surgical steps to be followed, and any related critical issues.

The utilized material was entirely PSI (cutters, trays, and screws), obtained with CAD and CAM reconstructions, including the 3D model to simulate the definitive glenoid component for the best orientation.

In each case, samples of tissue were collected intraoperatively and sent to the laboratory for prompt histologic examination.

All patients underwent postoperative radiographs and serial clinical and radiological follow-up evaluations after being discharged.
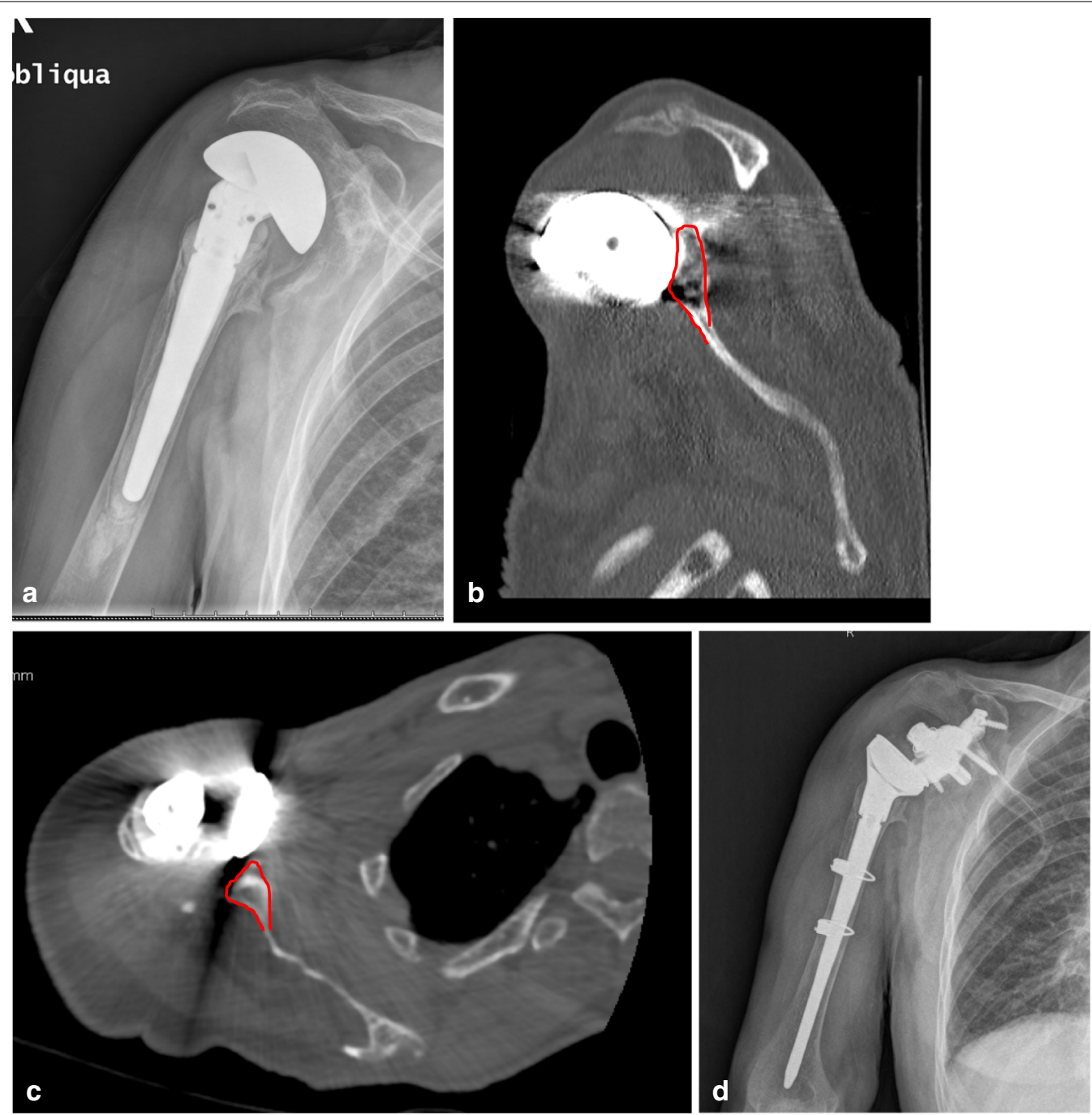

Fig. 2 a Preoperative X-rays, b preoperative sagittal plane CT scan, c preoperative oblique plane CT scan, d postoperative X-rays 
The Constant score, the ASES score and the pain VAS scale were used to determine the clinical outcomes. We observed an increased range of motion regarding the anterior and lateral elevation (mean increase $10.00 \pm 23.45$ and $10.00 \pm 25.39$, respectively). The difference between the internal and the external rotations showed no significant results statistically.

The mean pain reduction was $5.67 \pm 1.63$ according to the pain VAS scale. One patient complained of persistent pain at the end of the follow-up time.

The mean increase in the Constant score and the ASES score was $9.83 \pm 5.60$ and of $30.57 \pm 10.77$, respectively (Table 1).

The evaluation of radiolucent lines, signs of mobilization and potential glenoid notching was based on the radiological findings. None of the patients presented glenoid notching or implant mobilization. Two cases showed evidence of radiolucent lines $<2 \mathrm{~mm}$, which did not lead to changes or worsening of the clinical scores. No progression of such lines was evident until the end of the follow-up phase.

One female patient presented an episode of non-traumatic partial dislocation of the implant during the postoperative first trimester. The complete resolution of the correct implant stability was obtained with athletic taping and a physical therapy plan focused on rehabilitation and recovery of the correct shoulder mobility pattern.

\section{Discussion and review of the literature}

The limited number and the heterogeneity of the patients unquestionably complicate our observations and the preoperative studies based on the CT scan imaging (that guarantees a good resolution of shapes and planes) would not be sufficient to determine the areas with the best bone density. Furthermore, the time between the $\mathrm{CT}$ scan and the surgical procedure, approximately 3 months, could lead to additional bone and soft-tissue changes.

During the surgical procedures, the major difficulty we faced in all patients was the correct preparation of the portion of the remaining glenoid component in order to place the implant. We encountered anatomical changes, scar, and fibrous tissues because of the status post previous (often multiple) surgical procedure. Additionally, the placement of cut guides and trial components required a high degree of surgical expertise and perfectly accurate movements. The variation of a few grades when placing the PSI components, considering the presence of soft tissues not visualized with the CT scan, could cause a significant change in the orientation of the definitive component. Similar observations include the anchoring screws of the glenoid component; perforating in different directions compared to the planned direction might lead to positioning them in areas that do not guarantee correct primary stability of the implant.

The development of computer-aided surgery technologies and intraoperative navigation, recently introduced in shoulder replacement surgery, could probably be significantly useful and could overcome such intraoperative difficulties. Image-guided surgery and intraoperative feedback of the preoperative data and parameters are mandatory, particularly in difficult cases where accurate implant positioning is essential to obtain satisfactory results. Another focal point will be establishing a

Table 1 Summary of patient characteristics

\begin{tabular}{|c|c|c|c|c|c|c|c|c|c|c|c|}
\hline Name & Gender & Age & Classification (Antuna) & $\begin{array}{l}\text { Classification } \\
\text { (Seebauer) }\end{array}$ & on CS preop & CS & postop & ASES preop & ASES postop & VAS preop & VAS postop \\
\hline CM & F & 74 & Severe combined & E4 & 5 & 12 & & 10 & 25 & 8 & 5 \\
\hline OF & $\mathrm{F}$ & 66 & Severe combined & C4 & 13 & 17 & & 25 & 55 & 6 & 0 \\
\hline VG & $M$ & 71 & Severe combined & E4 & 18 & 38 & & 23.3 & 71.6 & 8 & 0 \\
\hline DBN & $M$ & 48 & Severe combined & E4 & 20 & 28 & & 13.3 & 40 & 8 & 2 \\
\hline $\mathrm{BN}$ & $\mathrm{F}$ & 56 & Severe combined & E4 & 18 & 26 & & 16.7 & 46.7 & 8 & 3 \\
\hline MW & $M$ & 69 & Severe combined & E4 & 16 & 28 & & 3.3 & 36.7 & 10 & 4 \\
\hline Name & $\begin{array}{l}\text { Ant. } \\
\text { elev. } \\
\text { preop }\end{array}$ & $\begin{array}{l}\text { Ant. } \\
\text { elev. } \\
\text { post }\end{array}$ & Abduction pre & $\begin{array}{l}\text { Abduction In } \\
\text { post }\end{array}$ & Intrarotation p & pre & Intraro & tation post & $\begin{array}{l}\text { Extrarotation } \\
\text { pre }\end{array}$ & $\begin{array}{l}\text { Extrarotation } \\
\text { post }\end{array}$ & $\begin{array}{l}\text { n Notching } \\
(y / n)\end{array}$ \\
\hline CM & $15^{\circ}$ & $25^{\circ}$ & $15^{\circ}$ & $25^{\circ}$ & Lateral thigh & & Lateral & thigh & $<10^{\circ}$ & $<10^{\circ}$ & y \\
\hline OF & $35^{\circ}$ & NA & $40^{\circ}$ & NA & Lateral thigh & & Lateral & thigh & $<10^{\circ}$ & NA & $n$ \\
\hline VG & $70^{\circ}$ & $90^{\circ}$ & $60^{\circ}$ & $80^{\circ}$ & Gluteus & & Gluteus & & $<10^{\circ}$ & $<10^{\circ}$ & $n$ \\
\hline DBN & $60^{\circ}$ & $70^{\circ}$ & $30^{\circ}$ & $50^{\circ}$ & Lateral thigh & & Lateral & thigh & $<10^{\circ}$ & $<10^{\circ}$ & $\mathrm{n}$ \\
\hline $\mathrm{BN}$ & $40^{\circ}$ & $65^{\circ}$ & $30^{\circ}$ & $50^{\circ}$ & -ateral thigh & & Lateral & thigh & $<10^{\circ}$ & $<10^{\circ}$ & $\mathrm{n}$ \\
\hline MW & $30^{\circ}$ & $60^{\circ}$ & $40^{\circ}$ & $70^{\circ}$ & -ateral thigh & & Lateral & thigh & $<10^{\circ}$ & $<10^{\circ}$ & $n$ \\
\hline
\end{tabular}

$N A$ not applicable, $y$ yes, $n$ no 
classification that allows the surgeon to select the correct custom implant based on bone loss morphology and extension. Eccentric and concentric defects require different solutions to obtain proper stability and fixation (Fig. 3a, b).

There are few studies in the literature regarding clinical experience with custom-made devices, and the outcomes, although encouraging, present a greatly reduced follow-up time.

A case report by Stoffelen et al. [32] concerning treatment with a glenoid custom-made anatomical total shoulder replacement, reported abduction of $90^{\circ}$, elevation of $110^{\circ}$, external rotation of $40^{\circ}$ and internal rotation at T12, with a Constant score of 51 at 2.5 years of follow-up.

A study by Chammaa et al. [1] in 37 patients treated with custom-made implants (CAD-CAM total shoulder replacement) for severe glenoid bone loss, obtained a postoperative pain level of 2.4. The mean active anterior elevation ranged from $39^{\circ}$ to $63^{\circ}$ and the external rotation from $6^{\circ}$ to $15^{\circ}$. During the 5 -year follow-up period, 6 of the 37 patients underwent an additional surgical procedure due to complications such as aseptic mobilization, fractures, and dislocation of the implant.
Shoulder - Defects C4
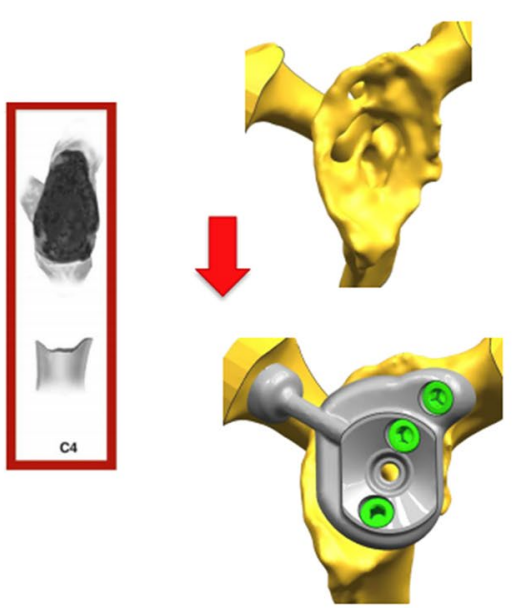

b

Shoulder - Defects E4
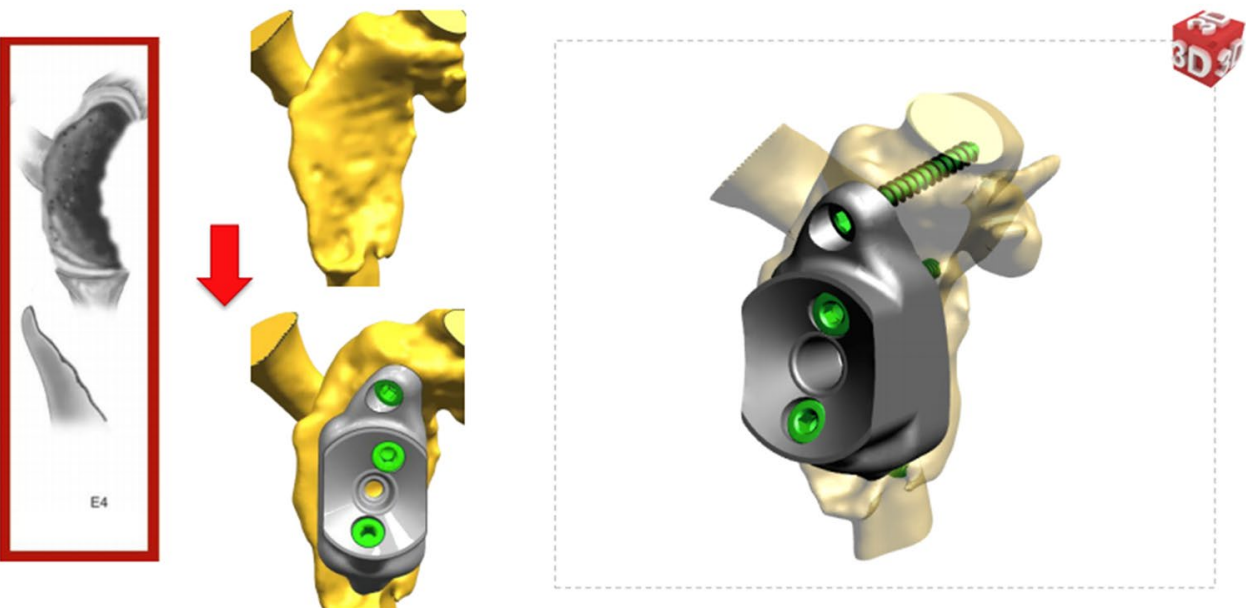

Fig. 3 a Defect C4 according to Seebauer classification and custom implant with coracoid fixation. b Defect E4 according to Seebauer classification and custom implant with posterior wedge 
Dines et al. reported the case of a patient treated with the patient-specific Glenoid Vault Reconstruction System developed by the authors in conjunction with Comprehensive Shoulder Arthroplasty System (Zimmer Biomet). After 18 months, the active range of motion of elevation forward was $130^{\circ}$ and $20^{\circ}$ for external rotation [33].

In a multicenter study by Debeer et al. of 10 patients treated with the Glenius Glenoid Reconstruction System, the mean patient-derived Constant-Murley score was $41.3 \pm 17.5$ points (range $18-76$ points) with a VAS scale of $3.3 \pm 2.5$ points (range $0-7$ points) at 30.5 months of follow-up [34].

In our study, we observed a slight improvement of the anterior and lateral elevation, possibly related to restoration of the center of rotation and to lateralization of the glenohumeral rotation center. This could have optimized the role of the deltoid for elevation of the limb, although with no improvement on the external rotation. The lack of internal rotation improvement could be related to degeneration of the subscapularis, further weakened by a new surgical access.

As well as the moderate increased range of motion, the patients were extremely satisfied with the reduction in perceived pain, a fundamental feature of the modest quality of life of patients before surgical treatment. Only one patient reported pain at the end of the followup period, although gradually reducing.

The dislocation reported in the postoperative period could be attributed to weakness of the anterior portion of the deltoid, in relation to the previous multiple surgical procedures. The recovery of stability consequent to the physical therapy appears to confirm this hypothesis.

The follow-up period is relatively brief and it is essential to monitor the above-mentioned changes in the future and possible evolution over time.

The radiological results were also very encouragingthe onset of radiolucent lines noted in two cases did not reflect pain-related symptoms, and did not negatively interfere with the clinical outcomes.

In fact, it is difficult to compare the planned images with the postoperative radiographs, although the directions of peg and screws are detectable (Fig. 4a, b).

Furthermore, the elevated costs related to the custom-made implant surgery require observation. The production of custom-made components and the work of the technicians unquestionably present certain advantages, although this technology is not applicable on a large scale.

This study does not aim to analyze the cost/benefits, and additional studies regarding this specific evaluation are required. Nevertheless, we can confirm that extremely accurate patient selection based on a patient's functional demand, pain-related symptoms, and motivation is required at the present time.

\section{Conclusions}

In our experience, the use of glenoid custom-made components based on CT scans results has been shown to be a potential solution for the management of severe glenoid bone loss, with encouraging clinical and radiological outcomes, although with a limited follow-up time.

These implants should be performed by dedicated surgeons with a high degree of experience in shoulder prosthesis, who are able to overcome unavoidable intraoperative difficulties.

The most reliable outcome of custom-made implants in severe glenoid bone loss is the decrease in pain. A large variety of outcomes were noted regarding functionality and, therefore, a significant clinical improvement is not guaranteed. Computer analysis of residual shape and amount of glenoid bone stock, in association with new classifications, could enable the surgeon to obtain good clinical and radiological outcomes, including the most difficult cases. 

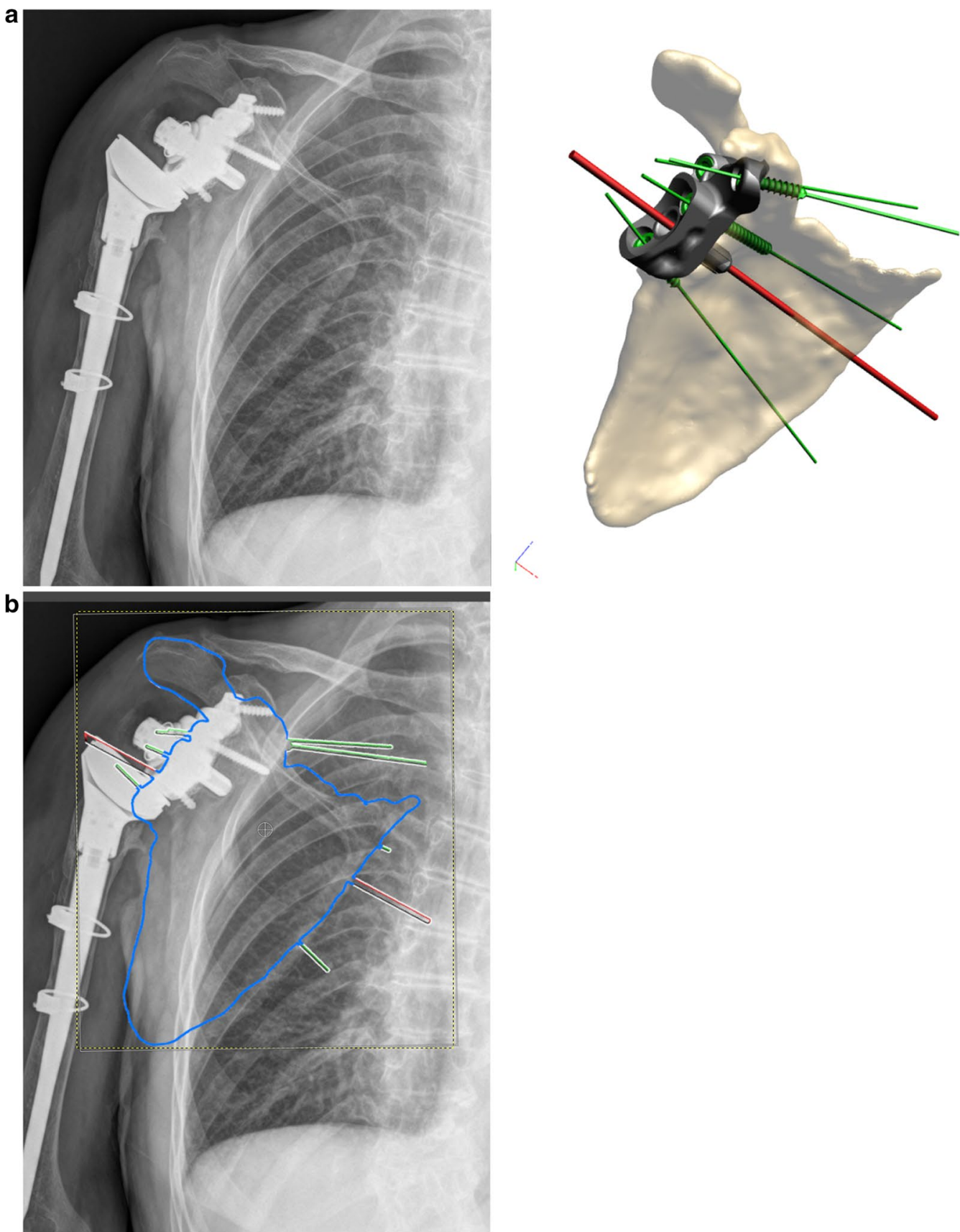

Fig. 4 a Comparison between postoperative X-rays and preoperative plan. b Matching between X-rays and preoperative plan with reconstruction of peg and screw directions

\section{Acknowledgements}

A special thanks to Fausto Sbaiz (LimaCorporate engineering) for the technical support and collaboration.

\section{Authors' contributions}

All authors read and approved the final manuscript.

\section{Funding}

No fundings.

\section{Availability of data and materials}

All data generated or analyzed during this study are included in this published article.

\section{Ethics approval and consent to participate}

The study has the approval from the ethics committee of the University of Modena and Reggio Emilia. 


\section{Consent for publication \\ Not applicable.}

\section{Competing interests}

Prof. G. Porcellini is a consultant for LimaCorporate with educational grants.

\section{Patient consent}

All patients gived their permission to use clinical informations and material in this review.

\section{Author details}

${ }^{1}$ Department of Orthopaedic Surgery, University of Modena and Reggio Emilia, Modena, Italy. ${ }^{2}$ Unit of Shoulder and Elbow Surgery, Ospedale Cervesi, Cattolica, RN, Italy.

Received: 21 February 2020 Accepted: 24 December 2020 Published online: 19 January 2021

\section{References}

1. Chammaa R, Uri O, Lambert S (2017) Primary shoulder arthroplasty using a custom-made hip-inspired implant for the treatment of advanced glenohumeral arthritis in the presence of severe glenoid bone loss. J Shoulder Elbow Surg 26(1):101-107

2. Lubbeke A, Rees JL, Barea C, Combescure C, Carr AJ, Silman AJ (2017) International variation in shoulder arthroplasty. Acta Orthoped 88(6):592-599

3. Kyriacou S, Khan S, Falworth M (2019) The management of glenoid bone loss in shoulder arthroplasty. J Arthrosc Joint Surg 6:21-30

4. Gupta A, Thussbas C, Koch M, Seebauer L (2017) Management of glenoid bone defects with reverse shoulder arthroplasty-surgical technique and clinical outcomes. J Shoulder Elbow Surg 27(5):853-862

5. Steinmann SP, Cofield RH (2000) Bone grafting for glenoid deficiency in total shoulder replacement. J Shoulder Elbow Surg 9:361-367

6. Gunther SB, Lynch TL (2012) Total shoulder replacement surgery with custom glenoid implants for severe bone deficiency. J Shoulder Elbow Surg 21:675-684

7. Denard PJ, Walch G (2013) Current concepts in the surgical management of primary glenohumeral arthritis with a biconcave glenoid. J Shoulder Elbow Surg 22:1589-1598

8. Cil A, Sperling JW, Cofield RH (2014) Nonstandard glenoid components for bone deficiencies in shoulder arthroplasty. J Shoulder Elbow Surg 23:e149-e157

9. Williams GR Jr, lannotti JP (2007) Options for glenoid bone loss: compos ites of prosthetics and biologics. J Shoulder Elbow Surg 16:S267-S272

10. Antuna SA, Sperling JW, Cofield RH, Rowland CM (2001) Glenoid revision surgery after total shoulder arthroplasty. J Shoulder Elbow Surg 10:217-224

11. Boileau P, Moineau G, Roussanne Y, O'Shea K (2017) Bony increased-offset reversed shoulder arthroplasty (BIO-RSA). JBJS Essent Surg Tech 7(4):e37

12. Boileau P, Moineau G, Roussanne Y, O'Shea K (2011) Bony increased-offset reversed shoulder arthroplasty: minimizing scapular impingement while maximizing glenoid fixation. Clin Orthop Relat Res 469(9):2558-2567

13. Paul RA, Maldonado-Rodriguez N, Docter S, Khan M, Veillette C, Verma N, Nicholson G, Leroux T (2019) Glenoid bone grafting in primary reverse total shoulder arthroplasty: a systematic review. J Shoulder Elbow Surg 22(11):1530-1536

14. Hendel MD, Werner BC, Camp CL, Gulotta LV, Walch G, Dines DM, Dines JS (2016) Management of the biconcave (B2) glenoid in shoulder arthroplasty: technical considerations. Am J Orthoped 45(4):220-227

15. lannotti JP, Lappin KE, Klotz CL, Reber EW, Swope SW (2013) Liftoff resistance of augmented glenoid components during cyclic fatigue loading in the posterior-superior direction. J Shoulder Elbow Surg 22(11):1530-1536
16. Sandow M, Schutz C (2016) Total shoulder arthroplasty using trabecular metal augments to address glenoid retroversion: the preliminary result of 10 patients with minimum 2-year follow-up. J Shoulder Elbow Surg 25(4):598-607

17. Hsu JE, Ricchetti ET, Huffman GR, lannotti JP, Glaser DL (2013) Addressing glenoid bone deficiency and asymmetric posterior erosion in shoulder arthroplasty. J Shoulder Elbow Surg 22:1298-1308

18. Iannotti JP, Norris TR (2003) Influence of preoperative factors on outcome of shoulder arthroplasty for glenohumeral osteoarthritis. J Bone Joint Surg Am 85:251-258

19. Levine WN, Djurasovic M, Glasson JM, Pollock RG, Flatow EL, Bigliani LU (1997) Hemiarthroplasty for glenohumeral osteoarthritis: results correlated to degree of glenoid wear. J Shoulder Elbow Surg 6:449-454

20. Levine WN, Fischer CR, Nguyen D, Flatow EL, Ahmad CS, Bigliani LU (2012) Long-term follow-up of shoulder hemiarthroplasty for glenohumeral osteoarthritis. J Bone Joint Surg Am 94:e164

21. Sperling JW, Cofield RH, Rowland CM (1998) Neer hemiarthroplasty and Neer total shoulder arthroplasty in patients fifty years old or less. Longterm results. J Bone Joint Surg Am 80:464-473

22. Strauss EJ, Roche C, Flurin PH, Wright T, Zuckerman JD (2009) The glenoid in shoulder arthroplasty. J Shoulder Elbow Surg 18:819-833

23. Hill JM, Norris TR (2001) Long-term results of total shoulder arthroplasty following bone-grafting of the glenoid. J Bone Joint Surg Am 83:877-883

24. Klika BJ, Wooten CW, Sperling JW, Steinmann SP, Schleck CD, Harmsen WS et al (2014) Structural bone grafting for glenoid deficiency in primary total shoulder arthroplasty. J Shoulder Elbow Surg 23:1066-1072

25. Nicholson GP, Cvetanovich GL, Rao AJ, O'Donnell P (2017) Posterior glenoid bone grafting in total shoulder arthroplasty for osteoarthritis with severe posterior glenoid wear. J Shoulder Elbow Surg 26:1844-1853

26. Sabesan V, Callanan M, Ho J, lannotti JP (2013) Clinical and radiographic outcomes of total shoulder arthroplasty with bone graft for osteoarthritis with severe glenoid bone loss. J Bone Joint Surg Am 95:1290-1296

27. Scalise JJ, lannotti JP (2008) Bone grafting severe glenoid defects in revision shoulder arthroplasty. Clin Orthop Relat Res 466:139-145

28. Frankle M, Siegal S, Pupello D, Saleem A, Mighell M, Vasey M (2005) The reverse shoulder prosthesis for glenohumeral arthritis associated with severe rotator cuff deficiency. A minimum two-year follow-up study of sixty patients. J Bone Joint Surg Am 87:1697-1705

29. Neyton L, Sirveaux F, Roche O, Mole D, Boileau P, Walch G (2004) Results of revision surgery for glenoid loosening: a multicentric series of 37 shoulder prosthesis [in French]. Rev Chir Orthop Reparatrice Appar Mot 90:111-121

30. Malhas A, Rashid A, Copas D, Bale S, Trail I (2019) Glenoid bone loss in primary and revision shoulder arthroplasty. Shoulder Elbow 8(4):229-240

31. Malhas AM, Granville-Chapman J, Robinson PM (2018) Reconstruction of the glenoid using autologous bone-graft and SMR Axioma TT metalbacked prosthesis: the first 45 sequential cases at a minimum of two years' follow-up. Bone Joint J 100-B(12):1609-1617

32. Stoffelen D, Eraly K, Debeer P (2015) The use of 3D printing technology in reconstruction of a severe glenoid defect: a case report with 25 years of follow-up. J Shoulder Elbow Surg 24:e218-e222

33. Dines DM, Gulotta L, Craig EV, Dines JS (2017) Novel solution for massive glenoid defects in shoulder arthroplasty: a patient-specific glenoid vault reconstruction system. Am J Orthop 46(2):104-108

34. Debeer P, Berghs B, Pouliart N, Van den Bogaert G, Verhaegen F, Nijs $S$ (2019) Treatment of severe glenoid deficiencies in reverse shoulder arthroplasty: the Glenius Glenoid Reconstruction System experience. J Shoulder Elbow Surg 28(8):1601-1608

\section{Publisher's Note}

Springer Nature remains neutral with regard to jurisdictional claims in published maps and institutional affiliations. 